\title{
Empa's New Clothes: The Untold Story of the Empa-Reg Outcome Trial
}

\author{
Aus Alzaid, MD
}

\begin{abstract}
It is no secret that the diabetes community has been yearning for good news for quite some time. One outstanding issue undermining effective diabetes management has been the lack of demonstrable effects of glycemic interventions on cardiovascular (CV) outcome in people with type 2 diabetes. This, however, seems to have changed recently after the publication of the Empa-Reg Outcome Trial. The study reported an impressive reduction in CV deaths that was observed within weeks of treatment with the diabetes agent, Empagliflozin. The results exceeded all expectations and was quickly embraced by a wide and receptive diabetes audience. Fans and admirers of the study have since set out to accommodate the new findings to reshape our diabetes practice guidelines. However, before everything becomes cast in stone, I believe there's another side to the story of the Empa-Reg Outcome trial that the diabetes community needs to hear. Here, I debate the merits of the study and present the case as to why I think we might be led down the garden path by accepting this study at mere face value.
\end{abstract}

Keywords: Type 2 DM, Cardiovascular complications, Empagliflozin, Clinical trial.

\section{Introduction}

D IABETES CARE HAS gone through a remarkable change over the past two decades as a result of the introduction of novel medications and technologies and the recent adoption of a patient-centered clinical approach. Treatment of cardiovascular $(\mathrm{CV})$ disease in people with diabetes has also become the focus of recent attention. Perhaps this should not be seen as surprising given the fact that nearly two thirds of patients with diabetes die from CV complications such as strokes and heart attacks. Moreover, evidence has shown that interventions aimed at controlling $\mathrm{CV}$ risk factors can successfully prevent $\mathrm{CV}$ complications ${ }^{1-3}$ and so treatment of hypertension and hyperlipidemia is now considered an integral part of everyday diabetes care. Unfortunately, the same could not be said about the impact of controlling hyperglycemia per se on $\mathrm{CV}$ outcome in patients with diabetes. ${ }^{4-6} \mathrm{~A}$ series of recent studies using a variety of glucose-lowering agents have failed to demonstrate any tangible benefits of glycemic control on CV outcome in people with diabetes. ${ }^{7-12}$ Fundamentally, hyperglycemia is supposed to be the hallmark of diabetes as a whole and so it was hard to understand how normalizing blood glucose could not prevent macro- vascular complications. This observation was hard to dismiss in clinical practice, especially when one considers the heavy burden of self-care expected on the part of the patient for control of hyperglycemia compared with the mere administration of a statin tablet needed to treat hyperlipidemia.

However, as we were all getting resigned to the idea of not to expect $\mathrm{CV}$ benefits through glycemic interventions, the news broke out and without warning from an unexpected corner-the Empa-Reg Outcome Study. This was a standard $\mathrm{CV}$ outcome trial, one of several studies imposed by the FDA on manufacturers to ensure $\mathrm{CV}$ safety of newly marketed diabetes drugs. Its findings were officially released at the annual European Association for the Study of Diabetes (EASD) meeting in Stockholm in September 2015. The study reported a staggering $38 \%$ reduction in $\mathrm{CV}$ mortality that was detectable within a short time of treatment with the diabetes agent, Empagliflozin. ${ }^{13}$ The diabetes community, thus, erupted with joy and excitement and given past failures, it felt like we had all won the lottery that day. Indeed, this looked like our brightest moment in diabetes care for decades. Opinion leaders and academic zealots sprang into action with their own speculations on the likely mechanisms in place to explain the startling results reported by the trial.

Department of Diabetes and Endocrinology, Prince Sultan Military Medical City, Riyadh, Saudi Arabia.

(C) Aus Alzaid, 2017; Published by Mary Ann Liebert, Inc. This Open Access article is distributed under the terms of the Creative Commons Attribution Noncommercial License (http://creativecommons.org/licenses/by-nc/4.0/) which permits any noncommercial use, distribution, and reproduction in any medium, provided the original author(s) and the source are credited. 
Words such as "magic bullet," "game-changer," and "holy grail" began to fly across professional diabetes sites and related literature. The diabetes scene looked set to change forever.

I was there at the EASD meeting to watch the drama unfold and like everyone else at the time, I was thrilled by the findings reported by the investigators of the Empa-Reg Outcome trial. No one had ever expected a diabetes drug to perform so well: an impressive $38 \%$ reduction in CV mortality.

However, from the outset, there has been an element of uncertainty, if not open disbelief, about the results reported by the Empa-Reg Outcome trial. Many of us in the diabetes community remain uneasy, although unspoken, about the inexplicable findings reported. To dispel any doubts, I decided to review the Empa-Reg Outcome study and explore the likely reasons behind the study's extraordinary results. Unfortunately, I did not find the peace of mind that I had originally set out to achieve. Here is my personal perspective on the reasons why I think we should all be concerned about the validity of the findings of the Empa-Reg outcome trial:

\section{Too good to be true}

It was the speed of divergence of the survival curves that seems to have caught everyone by surprise (Fig. 1): Within few short weeks of initiation of treatment, more patients within the placebo group began to die from $\mathrm{CV}$ disease than those taking the drug. This was completely unprecedented. In CV outcome trials, it takes $\sim 12-18$ months before the effect of intervention becomes apparent. ${ }^{14,15}$ Atherosclerosis, the pathological process that is responsible for CV disease, is meant to be chronic in nature, slow to develop, and hard to reverse. Unless the acute benefits seen were the result of a spurious chance, Empagliflozin must be operating in a league of its own, different from commonly used anti-ischemic therapies such as aspirin and statins.

The CV benefit with Empagliflozin seemed to also defy certain clinical perspectives: The outcome was uninfluenced by the renal status of the patient (high or low glomerular filtration rate) nor by the presence or absence of heart failure (in fact, greater benefits were seen in patients who did not have heart failure). Lastly, the profound reduction in CV outcome was seen on top of already optimal secondary prevention measures (for instance, more than $80 \%$ of the patients in the study were taking statin therapy).

Attempts made by some investigators to soothe and rationalize the unusual outcome of the Empa-Reg Outcome trial have not been successful. Intuitively, speculations advocating "diuresis" or some change in fuel substrate utilization as likely mechanisms are not convincing. ${ }^{16-18}$ Scientifically, such speculations lack supportive evidence and have been strongly rebuffed by other investigators. ${ }^{19,20}$

\section{The devil in the detail}

The difference in CV deaths between the two study groups was the main factor responsible for the observed difference in primary event rate (three-point MACE) in the Empa-Reg Outcome study. However, nearly $40 \%$ of the CV deaths reported in the trial were not $\mathrm{CV}$ in origin. These were nonassessable deaths that were presumed to be CV deaths because of lack of information about the actual cause of death and surrounding circumstances. Of relevance, the placebo group had proportionally more of those non-assessable deaths than did the intervention group. Critically, when these deaths (of uncertain cause) were removed from primary event analysis, Empagliflozin lost all superiority over placebo (HR $0.90,95 \%$ CL, $0.77-1.06) .^{21}$

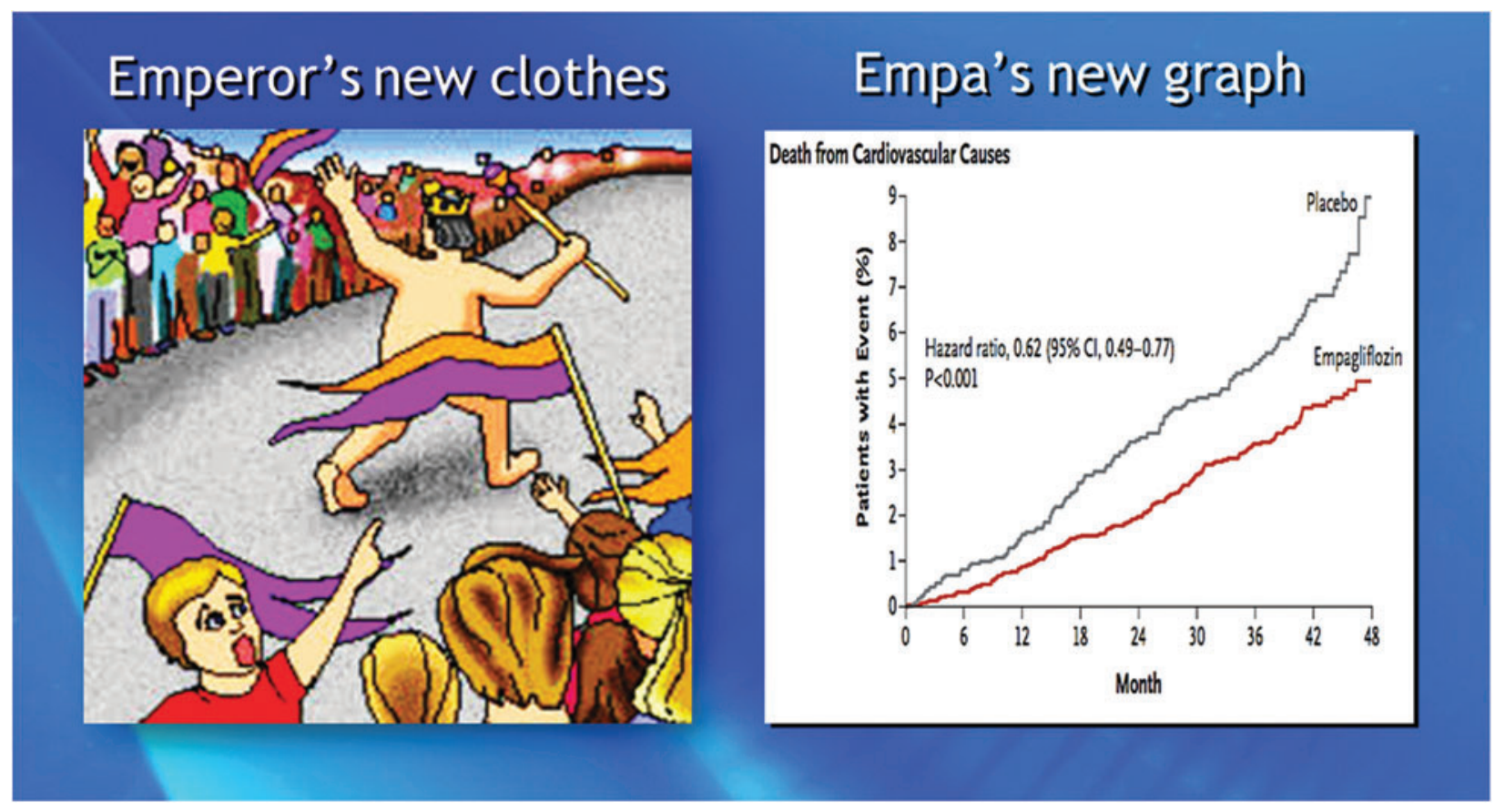

FIG. 1. Too good to be true! 
Table 1. Hazard Ratios for Cardiovascular Deaths PER REgion

\begin{tabular}{lccc}
\hline & $H R$ & $95 \% C I$ & $\mathrm{P}$ \\
\hline Europe & 0.72 & $0.51-1.01$ & 0.0552 \\
North America & 0.81 & $0.49-1.33$ & 0.3955 \\
Latin America & 0.43 & $0.24-0.77$ & 0.0045 \\
Asia & 0.35 & $0.19-0.65$ & 0.0008 \\
\hline
\end{tabular}

Another issue of concern is the wide geographic variation in $\mathrm{CV}$ deaths. ${ }^{22}$ Europe and North America (representing $61 \%$ of the global study population) showed no significant reduction in $\mathrm{CV}$ deaths with treatment. In fact, the only result found to reach statistical significance in Europe was a higher rate of stroke observed in the patients prescribed Empagliflozin. In sharp contrast, data from Asia and Latin America showed astonishingly positive CV results, thus tipping the overall balance of the trial in favor of Empagliflozin (Table 1). The reasons behind these contradictory results between regions are not clear and could reflect different local practices (such as the way in which causes of death had been adjudicated), variations in racial responses to treatment, or some yet unknown technical or logistical factors.

\section{Death seemed easier to prevent than illness}

Empagliflozin was shown to be highly effective in preventing death from $\mathrm{CV}$ disease. However, the drug had no effect on the individual components of $\mathrm{CV}$ disease, such as stroke and myocardial infarction, that patients had survived. Should not the two CV outcomes (CV death and CV complications) be moving in the same direction if treatment was meant to be targeting the same underlying disease? Conceptually, it is hard to understand how a drug can preferentially target $\mathrm{CV}$ complications that would only end in the death of the patient!

Data on stroke warrant extra mention. It was disappointing in the first place to observe that Empagliflozin did not alleviate the incidence of nonfatal stroke. It was baffling though to see how this could have happened while patients on Empagliflozin were enjoying significantly lower blood pressure than those in the control group. It remains to be explained, therefore, how patients prescribed Empagliflozin had numerically more strokes despite holding a double advantage of better glycemic control and lower blood pressure than the patients taking nothing (placebo).

\section{Lack of dose-response effect}

The presence of a direct dose-response relationship between a specific intervention and a certain clinical outcome likely indicates an underlying causal relationship between the two (e.g., number of cigarettes smoked and the relative risk of lung cancer). In the case of the Empa-Reg Outcome study, two different doses of Empagliflozin were prescribed, namely 10 and $25 \mathrm{mg}$ once daily. However, $\mathrm{CV}$ death rates were numerically comparable and survival curves were literally superimposable, irrespective of the dose used. If diuresis was the underlying mechanism claimed to prevent $\mathrm{CV}$ death, one would have expected a graded response in clinical outcome to the different "diuretic" doses used.

\section{Fringe benefits}

Professional statisticians are our trusted guardians who help us design clinical trials and carefully interpret the results afterward. In their books, what you have in mind at the outset of a clinical trial matters immensely to how you should interpret the final results. The Empa-Reg Outcome trial was a study that was designed specifically to test CV safety of Empagliflozin. This has been confirmed for Empagliflozin, with flying colors. Anything else found outside this primary objective, even if permitted to do so statistically, is technically of only limited significance - that is, it does not carry the same scientific weight of evidence, irrespective of the size of the effect found or the resultant $P$-value. Thus, the exploratory analysis reported to show a statistical difference in the incidence of heart failure between the study groups does not by itself establish proof of the clinical benefit of Empagliflozin over placebo. Rather, it serves as a convenient "tipoff" for the investigators to explore this avenue further (with a randomized controlled trial) if they want to confirm current results.

\section{What you see is not what you get}

The patient population studied in the Empa-Reg Outcome trial was a very unique group of elderly patients with diabetes who were at a very high risk of $\mathrm{CV}$ disease. In fact, more than $75 \%$ of the patients recruited had evidence of ischemic heart disease and nearly a quarter had already suffered from stroke, that is, a population severely crippled by advanced atherosclerotic disease. Contrast this with patients attending regular diabetes clinics, whereby on average less than $5 \%$ of the patients would report a past history of cerebrovascular accidents. In other words, what you see in the Empa-Reg Outcome trial is not necessarily what you get at your own clinic. Hence, we must resist all temptations to extrapolate the findings of the study to the wider diabetes population seen in everyday clinical practice.

\section{Conclusions: A Plea for Common Sense}

It has been said that there are two sides to every story. This could not be more appropriate than in the case of the interpretation of the Empa-Reg Outcome Trial.

The Empa-Reg Outcome study is undoubtedly an important and timely diabetes trial. It has spurred interest and raised hopes about the prospect of improving CV outcome for people with type 2 diabetes. However, as outlined earlier, the study has serious limitations that undermine the claims made of CV benefits. In fact, the prized finding of the study (Graph) looks conspicuously unreal and in my opinion, will always be impossible to explain biologically. Indeed, when I see everyone applauding this celebrity graph, I immediately think of the story line behind the traditional tale "The Emperor's New Clothes.',

I am also baffled by the decision of the FDA to approve the use of Empagliflozin for the prevention of $\mathrm{CV}$ deaths. Of note, the FDA decision has been taken against the advice of almost half of the advisory board members assembled to address the issue (11 vs. 12 had actually rejected the proposed application). This is not a game of sport to be settled by the net score achieved. If half the members of a medical jury were either in doubt of the evidence or had conflicting views 
with the other half, you can be sure that the final verdict itself would not be a sound one.

\section{Author Disclosure Statement}

No competing financial interests exist.

\section{References}

1. Gaede P, Lund-Andersen H, Parving HH, Pedersen O: effect of a multifactorial intervention on mortality in type 2 diabetes. N Engl J Med 2008;358:580-591.

2. ACCORD Study Group, Cushman WC, Evans GW, et al.: effects of intensive blood-pressure control in type 2 diabetes mellitus. N Engl J Med 2010;362:1575-1585.

3. Pyŏrälä K, Pedersen TR, Kjekshus J, et al.: Cholesterol lowering with simvastatin improves prognosis of diabetic patients with coronary heart disease. A subgroup analysis of the Scandinavian Simvastatin Survival Study (4S). Diabetes Care 1997;20:614-620.

4. Action to Control Cardiovascular Risk in Diabetes Study Group: Effects of intensive glucose lowering in type 2 diabetes. N Engl J Med 2008;358:2545-2559.

5. ADVANCE Collaborative Group; Patel A, MacMahon S, et al.: Intensive blood glucose control and vascular outcomes in patients with type 2 diabetes. $\mathrm{N}$ Engl $\mathrm{J}$ Med 2008;358:2560-2572.

6. Duckworth W, Abraira C, Moritz T, et al.; VADT Investigators: Glucose control and vascular complications in veterans with type 2 diabetes. N Engl J Med 2009;360: 129-139.

7. Nissen SE, Wolski K: Effect of rosiglitazone on the risk of myocardial infarction and death from cardiovascular causes. N Engl J Med 2007;356:2457-2471.

8. White WB, Cannon CP, Heller SR, et al.; EXAMINE Investigators: Alogliptin after acute coronary syndrome in patients with type 2 diabetes. N Engl J Med 2013;369: 1327-1335.

9. Scirica BM, Bhatt DL, Braunwald E, et al.; SAVOR-TIMI 53 Steering Committee and Investigators: Saxagliptin and cardiovascular outcomes in patients with type 2 diabetes mellitus. N Engl J Med 2013;369:1317-1326.

10. Green JB, Bethel MA, Armstrong PW, et al.; TECOS Study Group: Effect of sitagliptin on cardiovascular outcomes in type 2 diabetes. N Engl J Med 2015;373:232-242.

11. Pfeffer MA, Claggett B, Diaz R, et al.: Lixisenatide in patients with type 2 diabetes and acute coronary syndrome. N Engl J Med 2015;373:2247-2257.
12. ORIGIN Trial Investigators; Gerstein HC, Bosch J, Dagenais GR, et al.: Basal insulin and cardiovascular and other outcomes in dysglycemia. N Engl J Med 2012;367: 319-328.

13. Zinman B, Wanner C, Lachin JM, et al.; EMPA-REG OUTCOME Investigators. Empagliflozin, cardiovascular outcomes, and mortality in Type 2 diabetes. N Engl J Med 2015;373:2117-2128.

14. The Scandinavian Simvastatin Survival Study Group: Randomized trial of cholesterol lowering in 4444 patients with coronary heart disease: the Scandinavian Simvastatin Survival Study (4S). Lancet 1994;344:1383-1389.

15. Marso SP, Daniels GH, Brown-Frandsen K, et al.; LEADER Trial Investigators. Liraglutide and cardiovascular outcomes in Type 2 diabetes. N Engl J Med 2016;375: 311-322.

16. DeFronzo RA, McMurray J: EMPA-REG-The "diuretic hypothesis". J Diabetes Complications 2015; [Epub ahead of print]. doi:10.1016/j.jdiacomp.2015.10.012.

17. Ferrannini E, Mark M, Mayoux E: CV Protection in the EMPA-REG OUTCOME Trial: A "Thrifty Substrate" hypothesis. Diabetes Care 2016;39:1108-1114.

18. Mudaliar S, Alloju S, Henry RR: Can a shift in fuel energetics explain the beneficial cardiorenal outcomes in the EMPA-REG OUTCOME study? A unifying hypothesis. Diabetes Care 2016;39:1115-1122.

19. Scheen AJ: Reappraisal of the diuretic effect of empagliflozin in the EMPA-REG OUTCOME trial: comparison with classic diuretics. Diabetes Metab 2016;42:224-233.

20. Lopaschuk GD, Verma S: Empagliflozin's fuel hypothesis: not so soon. Cell Metab 2016;24:200-202.

21. www.fda.gov/downloads/advisorycommittees/committees meetingmaterials/drugs/endocrinologicandmetabolicdrugs advisorycommittee/ucm508422.pdf (accessed January 13, 2017).

22. www.iqwig.de/download/A16-12_Empagliflozin_Extractof-dossier-assessment.pdf (accessed January 13, 2017).

Address correspondence to: Aus Alzaid, MD Department of Diabetes and Endocrinology Prince Sultan Military Medical City Riyadh, Saudi Arabia

E-mail: drausalzaid@hotmail.com 